[Ni(1)]; $S=0$.

61

$\mathrm{XYZ}$ file generated by orca_plot on BaseName=HS

$\begin{array}{llll}\mathrm{Ni} & 5.681542 & 4.223084 & 7.137250 \\ \mathrm{O} & 6.941207 & 5.410691 & 6.455006 \\ \mathrm{O} & 5.914522 & 5.169349 & 8.723907 \\ \mathrm{~N} & 4.435329 & 3.061842 & 7.848725\end{array}$

$\begin{array}{llll}\text { O } & 7.283664 & 3.874448 & 1.958901\end{array}$

$\begin{array}{llll}\mathrm{O} & 2.826557 & 2.939026 & 11.582513\end{array}$

$\begin{array}{llll}\text { N } & 5.485712 & 3.300689 & 5.550448\end{array}$

$\begin{array}{llll}\text { C } & 4.018159 & 2.057109 & 6.947674\end{array}$

C $\quad 5.297919 \quad 5.061371 \quad 9.845816$

$\begin{array}{llll}\text { C } & 3.929242 & 3.152909 & 9.067787\end{array}$

$\begin{array}{llll}\text { H } & 3.158806 & 2.433378 & 9.358729\end{array}$

$\begin{array}{llll}\text { C } & 4.607287 & 2.197487 & 5.641104\end{array}$

C $7.404716 \quad 5.529505 \quad 5.262469$

$\begin{array}{llll}\text { C } & 6.084067 & 3.617239 & 4.414076\end{array}$

$\begin{array}{llll}\text { H } & 5.868026 & 3.006980 & 3.532908\end{array}$

$\begin{array}{llll}\text { C } & 6.990392 & 4.672881 & 4.198984\end{array}$

C $4.272773 \quad 4.092109 \quad 10.058264$

$\begin{array}{llll}\text { C } & 3.157304 & 1.015763 & 7.223560\end{array}$

$\begin{array}{llll}\text { H } & 2.709751 & 0.869131 & 8.205364\end{array}$

$\begin{array}{llll}\text { C } & 3.493303 & 3.939444 & 11.311432\end{array}$

$\begin{array}{llll}\text { C } & 2.830365 & 0.078060 & 6.207749\end{array}$

$\begin{array}{llll}\text { C } & 4.313901 & 1.299187 & 4.636813\end{array}$

H $4.746236 \quad 1.369737 \quad 3.639913$

$\begin{array}{llll}\text { C } & 7.463075 & 4.762007 & 2.795390\end{array}$

$\begin{array}{llll}\text { C } & 3.418485 & 0.224959 & 4.885608\end{array}$

$\begin{array}{llll}\text { C } & 8.431816 & 6.619474 & 5.122897\end{array}$

$\begin{array}{llll}\text { C } & 5.790762 & 6.016762 & 10.897988\end{array}$

$\begin{array}{llll}\text { O } & 3.551059 & 5.018126 & 12.121913\end{array}$

C $\quad 2.832799 \quad 4.915915 \quad 13.394177$

$\begin{array}{llll}\text { O } & 8.088511 & 5.922100 & 2.500183\end{array}$

[Ni(1)]; $S=1$.

61

Coordinates from ORCA-job HS

$\begin{array}{lccc}\mathrm{Ni} & 5.918719 & 4.146835 & 7.242353 \\ \mathrm{O} & 7.275981 & 5.304567 & 6.458424 \\ \mathrm{O} & 6.148829 & 5.021785 & 8.967984 \\ \mathrm{~N} & 4.539953 & 2.993649 & 7.938027 \\ \mathrm{O} & 7.364444 & 3.857936 & 1.922073 \\ \mathrm{O} & 2.787653 & 2.950859 & 11.632007 \\ \mathrm{~N} & 5.636360 & 3.232688 & 5.569234 \\ \mathrm{C} & 4.112214 & 2.037012 & 6.999996 \\ \mathrm{C} & 5.469009 & 4.898989 & 10.046113 \\ \mathrm{C} & 3.953457 & 3.126628 & 9.119822 \\ \mathrm{H} & 3.079677 & 2.507257 & 9.352071 \\ \mathrm{C} & 4.717698 & 2.172464 & 5.681642 \\ \mathrm{C} & 7.679961 & 5.394005 & 5.246687 \\ \mathrm{C} & 6.163764 & 3.580471 & 4.403709 \\ \mathrm{H} & 5.848712 & 3.050492 & 3.498145 \\ \mathrm{C} & 7.114897 & 4.599774 & 4.191558 \\ \mathrm{C} & 4.337668 & 4.018689 & 10.141954 \\ \mathrm{C} & 3.230667 & 1.007185 & 7.252224 \\ \mathrm{H} & 2.783374 & 0.859337 & 8.234632 \\ \mathrm{C} & 3.458307 & 3.941002 & 11.339301 \\ \mathrm{C} & 2.881368 & 0.079024 & 6.231393\end{array}$

$\begin{array}{lccc}\text { C } & 8.603266 & 6.053617 & 1.135388 \\ \text { C } & 2.288112 & -1.650116 & 4.175837 \\ \text { C } & 1.704701 & -1.799444 & 5.496902 \\ \text { C } & 0.813920 & -2.885984 & 5.741335 \\ \text { H } & 0.385015 & -2.984288 & 6.739684 \\ \text { C } & 0.514368 & -3.774721 & 4.733849 \\ \text { C } & 1.084771 & -3.625937 & 3.434263 \\ \text { C } & 1.950892 & -2.592149 & 3.159391 \\ \text { H } & 2.396271 & -2.463907 & 2.171613 \\ \text { H } & 0.827449 & -4.344134 & 2.654203 \\ \text { H } & -0.167992 & -4.604666 & 4.924288 \\ \text { N } & 3.138071 & -0.640916 & 3.891919 \\ \text { N } & 1.986417 & -0.932935 & 6.492744 \\ \text { H } & 3.198846 & 4.028351 & 13.929022 \\ \text { H } & 1.764588 & 4.775234 & 13.177397 \\ \text { C } & 3.096840 & 6.194918 & 14.160315 \\ \text { H } & 4.168866 & 6.319160 & 14.365835 \\ \text { H } & 2.564145 & 6.157094 & 15.120445 \\ \text { H } & 2.738089 & 7.071326 & 13.602769 \\ \text { H } & 7.758681 & 5.968246 & 0.437532 \\ \text { H } & 9.296806 & 5.222939 & 0.945303 \\ \text { C } & 9.286421 & 7.401473 & 1.043298 \\ \text { H } & 8.580196 & 8.217791 & 1.248524 \\ \text { H } & 9.682043 & 7.536116 & 0.026564 \\ \text { H } & 10.124537 & 7.470339 & 1.750217 \\ \text { H } & 6.713075 & 6.485507 & 10.537475 \\ \text { H } & 5.969183 & 5.507069 & 11.852032 \\ \text { H } & 5.035953 & 6.792462 & 11.087433 \\ \text { H } & 9.337383 & 6.252465 & 4.624721 \\ \text { H } & 8.674845 & 6.999914 & 6.120869 \\ \text { H } & 8.037456 & 7.436787 & 4.503257\end{array}$




$\begin{array}{llll}\text { H } & 1.563924 & 4.875983 & 12.979291 \\ \mathrm{C} & 2.770123 & 6.421099 & 13.935477 \\ \mathrm{H} & 3.817493 & 6.600307 & 14.214224 \\ \mathrm{H} & 2.160918 & 6.448844 & 14.849531 \\ \mathrm{H} & 2.435052 & 7.231931 & 13.273755 \\ \mathrm{H} & 7.496146 & 6.023486 & 0.405424 \\ \mathrm{H} & 9.132915 & 5.455333 & 0.825194 \\ \mathrm{C} & 8.876105 & 7.617196 & 0.963204 \\ \mathrm{H} & 8.092421 & 8.343420 & 1.219749\end{array}$

\section{[Ni(1)(py)]; $S=1$.}

$$
72
$$

XYZ file generated by orca_plot on BaseName=HS

$\begin{array}{llll}\mathrm{Ni} & 6.145762 & 3.931739 & 7.333495 \\ \mathrm{O} & 7.459734 & 5.183984 & 6.495860 \\ \mathrm{O} & 6.241034 & 4.945550 & 9.055209 \\ \mathrm{~N} & 4.499220 & 3.027255 & 7.925634 \\ \mathrm{O} & 7.209515 & 3.978684 & 1.892701 \\ \mathrm{O} & 2.546846 & 3.143728 & 11.477725 \\ \mathrm{~N} & 5.621360 & 3.243627 & 5.562422 \\ \mathrm{C} & 4.045338 & 2.101699 & 6.977946 \\ \mathrm{C} & 5.470856 & 4.902814 & 10.072133 \\ \mathrm{C} & 3.873616 & 3.231242 & 9.068195 \\ \mathrm{H} & 2.926908 & 2.708562 & 9.250464 \\ \mathrm{C} & 4.665642 & 2.225050 & 5.662507 \\ \mathrm{C} & 7.765748 & 5.338674 & 5.266704 \\ \mathrm{C} & 6.106243 & 3.646898 & 4.404141 \\ \mathrm{H} & 5.698301 & 3.214679 & 3.482578 \\ \mathrm{C} & 7.114641 & 4.620483 & 4.202649 \\ \mathrm{C} & 4.282849 & 4.089628 & 10.118106 \\ \mathrm{C} & 3.130470 & 1.094878 & 7.218069 \\ \mathrm{H} & 2.684892 & 0.950482 & 8.202159 \\ \mathrm{C} & 3.341655 & 4.063350 & 11.263784 \\ \mathrm{C} & 2.752698 & 0.193358 & 6.188722 \\ \mathrm{C} & 4.326479 & 1.342057 & 4.655164 \\ \mathrm{H} & 4.795457 & 1.386345 & 3.672552 \\ \mathrm{C} & 7.421442 & 4.823770 & 2.766171 \\ \mathrm{C} & 3.361852 & 0.323895 & 4.873115 \\ \mathrm{C} & 8.893823 & 6.312042 & 5.019755 \\ \mathrm{C} & 5.926907 & 5.750366 & 11.235951 \\ \mathrm{O} & 3.393661 & 5.165868 & 12.047373 \\ \mathrm{C} & 2.526824 & 5.168940 & 13.225559 \\ \mathrm{O} & 7.918847 & 6.049700 & 2.477706 \\ \mathrm{C} & 8.286745 & 6.289522 & 1.082059 \\ \mathrm{C} & 2.131253 & -1.474839 & 4.127990 \\ \mathrm{C} & 1.529120 & -1.608791 & 5.440636 \\ \mathrm{C} & 0.574488 & -2.644128 & 5.661286 \\ \mathrm{H} & 0.131994 & -2.731447 & 6.654892\end{array}$

\section{[Ni(1)(py) $)_{2} ; s=1$.}

83

Coordinates from ORCA-job HS

$\begin{array}{llll}\mathrm{Ni} & 6.277785 & 3.881143 & 7.391290 \\ \mathrm{O} & 7.884886 & 4.805545 & 6.524569 \\ \mathrm{O} & 6.567983 & 4.546276 & 9.303909 \\ \mathrm{~N} & 4.644048 & 2.909086 & 7.986500 \\ \mathrm{O} & 6.724951 & 4.497161 & 1.900605 \\ \mathrm{O} & 2.291759 & 3.652270 & 11.237617\end{array}$

$\begin{array}{cccc}\mathrm{H} & 9.199504 & 7.811049 & -0.069434 \\ \mathrm{H} & 9.736545 & 7.772777 & 1.628129 \\ \mathrm{H} & 7.004348 & 6.017034 & 10.991634 \\ \mathrm{H} & 5.933573 & 5.156723 & 12.154157 \\ \mathrm{H} & 5.355514 & 6.611660 & 11.339372 \\ \mathrm{H} & 9.564852 & 5.970374 & 4.329977 \\ \mathrm{H} & 9.274772 & 6.573751 & 6.000508 \\ \mathrm{H} & 8.434772 & 7.299384 & 4.600623\end{array}$

$\begin{array}{llll}\mathrm{N} & 5.771165 & 3.123977 & 5.619214 \\ \mathrm{C} & 4.209467 & 1.978010 & 7.046001 \\ \mathrm{C} & 5.672001 & 4.677279 & 10.197575 \\ \mathrm{C} & 3.936453 & 3.244550 & 9.043509 \\ \mathrm{H} & 2.918399 & 2.846265 & 9.146101 \\ \mathrm{C} & 4.833889 & 2.099187 & 5.729646 \\ \mathrm{C} & 7.989160 & 5.138120 & 5.300317 \\ \mathrm{C} & 6.113535 & 3.659639 & 4.467594\end{array}$




$\begin{array}{llll}\text { H } & 5.578133 & 3.349997 & 3.560687 \\ \text { C } & 7.102837 & 4.656706 & 4.268195 \\ \text { C } & 4.344294 & 4.123586 & 10.079736 \\ \text { C } & 3.298890 & 0.966958 & 7.288093 \\ \text { H } & 2.857174 & 0.828797 & 8.275313 \\ \text { C } & 3.268105 & 4.387904 & 11.057377 \\ \text { C } & 2.930220 & 0.058040 & 6.260844 \\ \text { C } & 4.506884 & 1.207259 & 4.725713 \\ \text { H } & 4.988716 & 1.252993 & 3.748915 \\ \text { C } & 7.150834 & 5.134035 & 2.870104 \\ \text { C } & 3.546558 & 0.184557 & 4.945045 \\ \text { C } & 9.164229 & 6.039369 & 4.993589 \\ \text { C } & 6.128296 & 5.410384 & 11.439735 \\ \text { O } & 3.411468 & 5.562151 & 11.722902 \\ \text { C } & 2.417278 & 5.848043 & 12.754531 \\ \text { O } & 7.663217 & 6.382979 & 2.736329 \\ \text { C } & 7.787961 & 6.891846 & 1.372229 \\ \text { C } & 2.326502 & -1.625330 & 4.204439 \\ \text { C } & 1.718606 & -1.754786 & 5.513283 \\ \text { C } & 0.767109 & -2.792316 & 5.733846 \\ \text { H } & 0.319521 & -2.876287 & 6.725581 \\ \text { C } & 0.430289 & -3.654817 & 4.713004 \\ \text { C } & 1.024849 & -3.524784 & 3.424280 \\ \text { C } & 1.951623 & -2.536039 & 3.174400 \\ \text { H } & 2.416880 & -2.421933 & 2.193788 \\ \text { H } & 0.739325 & -4.218051 & 2.631484 \\ \text { H } & -0.300337 & -4.446569 & 4.887091 \\ \text { N } & 3.236872 & -0.659546 & 3.942308 \\ \text { N } & 2.033663 & -0.911912 & 6.523762 \\ \text { H } & 2.404905 & 5.014046 & 13.470908 \\ \text { H } & 1.427624 & 5.907915 & 12.280187 \\ \text { C } & 2.813778 & 7.154859 & 13.409624 \\ \text { H } & 3.806237 & 7.079175 & 13.875048 \\ \text { H } & 2.083770 & 7.404476 & 14.192947 \\ \text { H } & 2.828114 & 7.975405 & 12.678388 \\ \text { H } & \end{array}$

\section{[Ni(1)(CN-py)]; $S=1$.}

73

Coordinates from ORCA-job HS

$\begin{array}{cccc}\mathrm{Ni} & 6.151116 & 3.934211 & 7.331663 \\ \mathrm{O} & 7.459288 & 5.189863 & 6.494641 \\ \mathrm{O} & 6.249146 & 4.945877 & 9.052435 \\ \mathrm{~N} & 4.509629 & 3.022870 & 7.926774 \\ \mathrm{O} & 7.220618 & 3.975290 & 1.894352 \\ \mathrm{O} & 2.579399 & 3.121410 & 11.492375 \\ \mathrm{~N} & 5.625773 & 3.244680 & 5.561464 \\ \mathrm{C} & 4.054128 & 2.098180 & 6.978098 \\ \mathrm{C} & 5.480043 & 4.901814 & 10.070994 \\ \mathrm{C} & 3.887843 & 3.223163 & 9.072418 \\ \mathrm{H} & 2.944094 & 2.696358 & 9.257389 \\ \mathrm{C} & 4.670923 & 2.224442 & 5.661909 \\ \mathrm{C} & 7.763811 & 5.346284 & 5.264643 \\ \mathrm{C} & 6.111307 & 3.648040 & 4.403180 \\ \mathrm{H} & 5.705621 & 3.213381 & 3.481926 \\ \mathrm{C} & 7.117032 & 4.623870 & 4.201693 \\ \mathrm{C} & 4.297420 & 4.082363 & 10.121015 \\ \mathrm{C} & 3.140325 & 1.090668 & 7.218897 \\ \mathrm{H} & 2.696857 & 0.944621 & 8.203648 \\ \mathrm{C} & 3.360887 & 4.050272 & 11.271351\end{array}$

$\begin{array}{llll}\mathrm{H} & 6.787259 & 6.927618 & 0.919165 \\ \mathrm{H} & 8.403870 & 6.190970 & 0.790804 \\ \mathrm{C} & 8.419999 & 8.265754 & 1.463951 \\ \mathrm{H} & 7.796196 & 8.947795 & 2.058491 \\ \mathrm{H} & 8.523566 & 8.685762 & 0.453206 \\ \mathrm{H} & 9.418317 & 8.212373 & 1.919027 \\ \mathrm{H} & 7.225299 & 5.401580 & 11.452248 \\ \mathrm{H} & 5.733593 & 4.968840 & 12.362025 \\ \mathrm{H} & 5.781813 & 6.453233 & 11.409497 \\ \mathrm{H} & 9.664970 & 5.780094 & 4.053577 \\ \mathrm{H} & 9.868701 & 5.972226 & 5.831990 \\ \mathrm{H} & 8.821911 & 7.079792 & 4.897379 \\ \mathrm{~N} & 7.464485 & 2.187468 & 7.805745 \\ \mathrm{C} & 7.286313 & 1.493501 & 8.947265 \\ \mathrm{H} & 6.611773 & 1.938774 & 9.678203 \\ \mathrm{C} & 8.284550 & 1.680287 & 6.864157 \\ \mathrm{H} & 8.395066 & 2.273193 & 5.956260 \\ \mathrm{C} & 7.917739 & 0.275043 & 9.189404 \\ \mathrm{C} & 8.953711 & 0.468724 & 7.027707 \\ \mathrm{C} & 8.764183 & -0.252109 & 8.210146 \\ \mathrm{H} & 7.739386 & -0.250267 & 10.127728 \\ \mathrm{H} & 9.604560 & 0.098509 & 6.235469 \\ \mathrm{H} & 9.267183 & -1.207098 & 8.365627 \\ \mathrm{~N} & 5.183025 & 5.654926 & 7.034171 \\ \mathrm{C} & 3.972585 & 5.639582 & 6.444905 \\ \mathrm{H} & 3.579999 & 4.659450 & 6.173231 \\ \mathrm{C} & 5.713672 & 6.841009 & 7.390426 \\ \mathrm{H} & 6.694439 & 6.799763 & 7.863526 \\ \mathrm{C} & 3.249036 & 6.802297 & 6.187976 \\ \mathrm{C} & 5.055892 & 8.050063 & 7.169357 \\ \mathrm{C} & 3.800292 & 8.032488 & 6.556393 \\ \mathrm{H} & 2.272759 & 6.737903 & 5.708344 \\ \mathrm{H} & 5.524578 & 8.985625 & 7.474156 \\ \mathrm{H} & 3.261051 & 8.961793 & 6.368697\end{array}$

$\begin{array}{llll}\text { C } & 2.760645 & 0.191270 & 6.188518 \\ \text { C } & 4.330015 & 1.343638 & 4.653370 \\ \text { H } & 4.796126 & 1.390505 & 3.669555 \\ \text { C } & 7.425724 & 4.824992 & 2.764512 \\ \text { C } & 3.366549 & 0.324598 & 4.871990 \\ \text { C } & 8.884643 & 6.327217 & 5.017600 \\ \text { C } & 5.932895 & 5.755735 & 11.230792 \\ \text { O } & 3.402393 & 5.157562 & 12.047681 \\ \text { C } & 2.538254 & 5.158331 & 13.228393 \\ \text { O } & 7.915347 & 6.052794 & 2.473790 \\ \text { C } & 8.282911 & 6.291757 & 1.077460 \\ \text { C } & 2.135204 & -1.472940 & 4.126087 \\ \text { C } & 1.535943 & -1.609598 & 5.440090 \\ \text { C } & 0.582015 & -2.645657 & 5.660960 \\ \text { H } & 0.141709 & -2.734980 & 6.655328 \\ \text { C } & 0.235347 & -3.498455 & 4.636592 \\ \text { C } & 0.821055 & -3.361426 & 3.343425 \\ \text { C } & 1.749002 & -2.375405 & 3.092173 \\ \text { H } & 2.207163 & -2.256438 & 2.108969 \\ \text { H } & 0.526396 & -4.048583 & 2.548777 \\ \text { H } & -0.496564 & -4.289121 & 4.809791 \\ \text { N } & 3.046284 & -0.510206 & 3.863965\end{array}$




$\begin{array}{cccc}\mathrm{N} & 1.861307 & -0.776450 & 6.452952 \\ \mathrm{H} & 2.780354 & 4.275964 & 13.837439 \\ \mathrm{H} & 1.494249 & 5.073571 & 12.895064 \\ \mathrm{C} & 2.796133 & 6.453196 & 13.970187 \\ \mathrm{H} & 3.843790 & 6.522874 & 14.293258 \\ \mathrm{H} & 2.157235 & 6.492201 & 14.863368 \\ \mathrm{H} & 2.561168 & 7.322808 & 13.340465 \\ \mathrm{H} & 7.385352 & 6.165931 & 0.456272 \\ \mathrm{H} & 9.020449 & 5.534079 & 0.777757 \\ \mathrm{C} & 8.840236 & 7.697226 & 0.994652 \\ \mathrm{H} & 8.093108 & 8.438219 & 1.311424 \\ \mathrm{H} & 9.119326 & 7.914585 & -0.046226 \\ \mathrm{H} & 9.735886 & 7.806095 & 1.621050 \\ \mathrm{H} & 6.962436 & 6.076134 & 11.035076 \\ \mathrm{H} & 5.877150 & 5.215882 & 12.183922 \\ \mathrm{H} & 5.289584 & 6.640620 & 11.329953\end{array}$

\section{[Ni(1)(CN-py) $)_{2}$; $S=1$.}

85

Coordinates from ORCA-job HS

$\begin{array}{llll}\mathrm{Ni} & 6.285069 & 3.891002 & 7.391415 \\ \mathrm{O} & 7.890267 & 4.819078 & 6.529768 \\ \mathrm{O} & 6.580735 & 4.558513 & 9.299066 \\ \mathrm{~N} & 4.655133 & 2.915697 & 7.987716 \\ \mathrm{O} & 6.748213 & 4.506015 & 1.902904 \\ \mathrm{O} & 2.315447 & 3.656185 & 11.250724 \\ \mathrm{~N} & 5.780288 & 3.132121 & 5.620210 \\ \mathrm{C} & 4.223128 & 1.980390 & 7.047921 \\ \mathrm{C} & 5.684130 & 4.688502 & 10.195034 \\ \mathrm{C} & 3.949942 & 3.250712 & 9.046913 \\ \mathrm{H} & 2.935259 & 2.845822 & 9.155283 \\ \mathrm{C} & 4.846351 & 2.102227 & 5.731968 \\ \mathrm{C} & 7.994486 & 5.151706 & 5.303551 \\ \mathrm{C} & 6.124444 & 3.668328 & 4.468997 \\ \mathrm{H} & 5.595206 & 3.353152 & 3.560540 \\ \mathrm{C} & 7.109329 & 4.669922 & 4.272865 \\ \mathrm{C} & 4.358152 & 4.134514 & 10.078327 \\ \mathrm{C} & 3.317538 & 0.966295 & 7.292737 \\ \mathrm{H} & 2.876536 & 0.828234 & 8.280230 \\ \mathrm{C} & 3.283155 & 4.399049 & 11.059931 \\ \mathrm{C} & 2.954144 & 0.053346 & 6.266759 \\ \mathrm{C} & 4.525061 & 1.207370 & 4.729539 \\ \mathrm{H} & 5.007084 & 1.253924 & 3.752941 \\ \mathrm{C} & 7.159316 & 5.149076 & 2.873567 \\ \mathrm{C} & 3.569911 & 0.179944 & 4.951614 \\ \mathrm{C} & 9.168364 & 6.053355 & 4.998044 \\ \mathrm{C} & 6.142197 & 5.421228 & 11.435665 \\ \mathrm{O} & 3.421172 & 5.580883 & 11.710338 \\ \mathrm{C} & 2.428166 & 5.873530 & 12.742395 \\ \mathrm{O} & 7.653077 & 6.404757 & 2.747224 \\ \mathrm{C} & 7.776115 & 6.921913 & 1.385249 \\ \mathrm{C} & 2.362054 & -1.639024 & 4.215284 \\ \mathrm{C} & 1.753975 & -1.768391 & 5.524546 \\ \mathrm{C} & 0.808801 & -2.811218 & 5.747936 \\ \mathrm{H} & 0.361224 & -2.895056 & 6.739619 \\ \mathrm{C} & 0.478611 & -3.678593 & 4.729456 \\ \mathrm{C} & 1.073279 & -3.548728 & 3.440466 \\ \mathrm{C} & 1.993771 & -2.555265 & 3.187619 \\ \mathrm{H} & 2.458932 & -2.441303 & 2.207018\end{array}$

$\begin{array}{cccc}\mathrm{H} & 9.605513 & 5.954350 & 4.279851 \\ \mathrm{H} & 9.384508 & 6.521310 & 5.973579 \\ \mathrm{H} & 8.484276 & 7.270801 & 4.621686 \\ \mathrm{~N} & 7.452926 & 2.490689 & 7.810184 \\ \mathrm{C} & 7.386625 & 1.889156 & 9.016130 \\ \mathrm{H} & 6.645443 & 2.274197 & 9.715282 \\ \mathrm{C} & 8.347957 & 2.052398 & 6.900606 \\ \mathrm{H} & 8.360831 & 2.564977 & 5.939368 \\ \mathrm{C} & 8.212284 & 0.827253 & 9.362681 \\ \mathrm{C} & 9.213339 & 0.996793 & 7.159310 \\ \mathrm{C} & 9.142159 & 0.368709 & 8.414358 \\ \mathrm{H} & 8.128304 & 0.365814 & 10.344776 \\ \mathrm{H} & 9.919954 & 0.668951 & 6.398982 \\ \mathrm{C} & 10.007518 & -0.725113 & 8.723415 \\ \mathrm{~N} & 10.713320 & -1.618051 & 8.975495\end{array}$


$\begin{array}{llll}H & 5.568489 & 8.995414 & 7.425407\end{array}$

C $9.350596-1.530090 \quad 8.339619$

$\begin{array}{llll}\text { N } & 9.863432 & -2.566916 & 8.487212\end{array}$

\section{[Ni(1)(DMAP)]; $S$ = 1.}

80

Coordinates from ORCA-job HS

$\mathrm{Ni} \quad 6.160349 \quad 3.934181$

$\begin{array}{llll}0 & 7.473292 & 5.197751 & 6.528343\end{array}$

$\begin{array}{llll}\text { O } & 6.256204 & 4.924171 & 9.097173\end{array}$

$\begin{array}{llll}N & 4.506996 & 3.032448 & 7.935016\end{array}$

$\begin{array}{llll}\mathrm{O} & 7.220606 & 4.039454 & 1.912409\end{array}$

$\begin{array}{llll}\text { O } & 2.479314 & 3.193280 & 11.446087\end{array}$

$\begin{array}{llll}\mathrm{N} & 5.631425 & 3.268363 & 5.576292\end{array}$

$\begin{array}{llll}\text { C } & 4.065920 & 2.105489 & 6.984502\end{array}$

$\begin{array}{llll}\text { C } & 5.469833 & 4.886842 & 10.101055\end{array}$

$\begin{array}{llll}\text { C } & 3.862732 & 3.244059 & 9.065399\end{array}$

$\begin{array}{llll}\text { H } & 2.905153 & 2.734511 & 9.228203\end{array}$

$\begin{array}{llll}\text { C } & 4.687690 & 2.239265 & 5.670399\end{array}$

$\begin{array}{llll}\text { C } & 7.780278 & 5.360474 & 5.301122\end{array}$

$\begin{array}{llll}\text { C } & 6.118127 & 3.678870 & 4.421670\end{array}$

$\begin{array}{llll}\text { H } & 5.713662 & 3.251377 & 3.496194\end{array}$

C $\quad 7.125662 \quad 4.655484 \quad 4.229750$

C $\quad 4.264492 \quad 4.097856 \quad 10.122212$

$\begin{array}{llll}\text { C } & 3.166845 & 1.083943 & 7.222701\end{array}$

$\begin{array}{llll}\text { H } & 2.725232 & 0.931299 & 8.207407\end{array}$

C $\quad 3.298041 \quad 4.095136 \quad 11.245592$

$\begin{array}{llll}\text { C } & 2.805158 & 0.177181 & 6.192143\end{array}$

C $4.362986 \quad 1.352545 \quad 4.661496$

H $4.834369 \quad 1.404159 \quad 3.680341$

$\begin{array}{llll}\text { C } & 7.432104 & 4.874209 & 2.796205\end{array}$

$\begin{array}{llll}\text { C } & 3.414337 & 0.318929 & 4.877099\end{array}$

$\begin{array}{llll}\text { C } & 8.915407 & 6.328399 & 5.061728\end{array}$

C $\quad 5.925047 \quad 5.712256 \quad 11.281852$

$\begin{array}{llll}\text { O } & 3.353130 & 5.201319 & 12.025215\end{array}$

C $2.460643 \quad 5.226299 \quad 13.183340$

$\begin{array}{llll}\text { O } & 7.928319 & 6.104296 & 2.521066\end{array}$

$\begin{array}{llll}\text { C } & 8.297414 & 6.359534 & 1.128809\end{array}$

$\begin{array}{llll}\text { C } & 2.216836 & -1.501522 & 4.129757\end{array}$

$\begin{array}{llll}\text { C } & 1.615117 & -1.646460 & 5.441026\end{array}$

$\begin{array}{llll}\text { C } & 0.679261 & -2.699004 & 5.659939\end{array}$

$\begin{array}{llll}\mathrm{H} & 0.236747 & -2.794449 & 6.652818\end{array}$

$\begin{array}{llll}\text { C } & 0.352062 & -3.560536 & 4.636027\end{array}$

$\begin{array}{llll}\text { C } & 0.940096 & -3.415309 & 3.345247\end{array}$

$\begin{array}{llll}\text { C } & 1.851122 & -2.412628 & 3.096196\end{array}$

H $\quad 2.311004 \quad-2.287095 \quad 2.114544$

\section{[Ni(1)(DMAP) $)_{2}$; $S=1$.}

99

Coordinates from ORCA-job HS

$\begin{array}{llll}\mathrm{Ni} & 6.317805 & 3.832029 & 7.516534 \\ \mathrm{O} & 7.935560 & 4.728037 & 6.565860 \\ \mathrm{O} & 6.609016 & 4.488674 & 9.432660 \\ \mathrm{~N} & 4.678826 & 2.870258 & 8.100034 \\ \mathrm{O} & 6.177438 & 5.031631 & 2.132768 \\ \mathrm{O} & 2.303981 & 3.653519 & 11.331988 \\ \mathrm{~N} & 5.789577 & 3.089185 & 5.735871 \\ \mathrm{C} & 4.282804 & 1.903932 & 7.181033\end{array}$

$\begin{array}{llll}\text { N } & 2.581317 & 10.248008 & 6.016844\end{array}$

$\begin{array}{llll}\text { C } & 3.147225 & 9.252599 & 6.237066\end{array}$

$\begin{array}{cccc}\mathrm{H} & 0.661480 & -4.109189 & 2.550580 \\ \mathrm{H} & -0.365899 & -4.364136 & 4.808290 \\ \mathrm{~N} & 3.112282 & -0.522916 & 3.869543 \\ \mathrm{~N} & 1.921946 & -0.805663 & 6.454173 \\ \mathrm{H} & 2.658386 & 4.335071 & 13.795557 \\ \mathrm{H} & 1.422747 & 5.176175 & 12.824762 \\ \mathrm{C} & 2.741200 & 6.509862 & 13.936701 \\ \mathrm{H} & 3.782202 & 6.544676 & 14.285919 \\ \mathrm{H} & 2.081819 & 6.566843 & 14.814012 \\ \mathrm{H} & 2.550370 & 7.388650 & 13.304770 \\ \mathrm{H} & 7.399414 & 6.248752 & 0.505284 \\ \mathrm{H} & 9.029833 & 5.601257 & 0.817994 \\ \mathrm{C} & 8.864673 & 7.762252 & 1.065212 \\ \mathrm{H} & 8.122744 & 8.503932 & 1.392561 \\ \mathrm{H} & 9.145298 & 7.992492 & 0.027514 \\ \mathrm{H} & 9.760956 & 7.856102 & 1.693156 \\ \mathrm{H} & 6.972132 & 5.991102 & 11.116867 \\ \mathrm{H} & 5.820974 & 5.169018 & 12.229033 \\ \mathrm{H} & 5.317832 & 6.623499 & 11.370302 \\ \mathrm{H} & 9.627644 & 5.955973 & 4.315382 \\ \mathrm{H} & 9.422554 & 6.500355 & 6.018270 \\ \mathrm{H} & 8.528391 & 7.284183 & 4.682214 \\ \mathrm{~N} & 7.441762 & 2.461837 & 7.808010 \\ \mathrm{C} & 7.379132 & 1.821082 & 8.998596 \\ \mathrm{H} & 6.667480 & 2.218257 & 9.722771 \\ \mathrm{C} & 8.307325 & 1.980903 & 6.885265 \\ \mathrm{H} & 8.333786 & 2.504483 & 5.929029 \\ \mathrm{C} & 8.153700 & 0.720624 & 9.311740 \\ \mathrm{C} & 9.121852 & 0.886305 & 7.103946 \\ \mathrm{C} & 9.071163 & 0.203518 & 8.352967 \\ \mathrm{H} & 8.037484 & 0.262955 & 10.291355 \\ \mathrm{H} & 9.783835 & 0.561715 & 6.304538 \\ \mathrm{~N} & 9.850275 & -0.877925 & 8.613571 \\ \mathrm{C} & 10.730032 & -1.416988 & 7.578191 \\ \mathrm{C} & 9.720636 & -1.588148 & 9.884872 \\ \mathrm{H} & 8.716193 & -2.025194 & 10.005097 \\ \mathrm{H} & 9.916219 & -0.915977 & 10.734373 \\ \mathrm{H} & 10.456349 & -2.397316 & 9.911825 \\ \mathrm{H} & 10.157102 & -1.788065 & 6.712728 \\ \mathrm{H} & 11.300776 & -2.249899 & 7.999520 \\ \mathrm{H} & 11.442277 & -0.654416 & 7.227628\end{array}$

$\begin{array}{llll}\text { C } & 5.702113 & 4.633452 & 10.311687 \\ \text { C } & 3.965843 & 3.205141 & 9.152320 \\ \text { H } & 2.952031 & 2.795517 & 9.256730 \\ \text { C } & 4.902535 & 2.027133 & 5.862316 \\ \text { C } & 7.860193 & 5.224546 & 5.395664 \\ \text { C } & 5.995677 & 3.722062 & 4.601637 \\ \text { H } & 5.386790 & 3.453977 & 3.727478 \\ \text { C } & 6.883353 & 4.812389 & 4.421436 \\ \text { C } & 4.368199 & 4.095492 & 10.181257 \\ \text { C } & 3.423118 & 0.854810 & 7.443737\end{array}$




\begin{tabular}{|c|c|c|c|}
\hline & 2.988928 & 0.715025 & 8.434139 \\
\hline & 3.286477 & 4.379815 & 11.145111 \\
\hline & 3.105038 & -0.094110 & 6.435202 \\
\hline & 4.618663 & 1.105539 & 4.873070 \\
\hline & 5.102342 & 1.160971 & 3.897295 \\
\hline & 6.652594 & 5.534755 & 3.156559 \\
\hline & 3.714830 & 0.036538 & 5.115238 \\
\hline & 8.922695 & 6.248711 & 5.065198 \\
\hline & 6.150769 & 5.362142 & 11.560726 \\
\hline & 3.430149 & 5.566633 & 11.790798 \\
\hline & 2.432274 & 5.874058 & 12.812071 \\
\hline & 6.929671 & 6.860327 & 3.247208 \\
\hline & 6.671133 & 7.667743 & 2.062000 \\
\hline & 2.599875 & -1.857817 & 4.419069 \\
\hline & 1.999067 & -1.990436 & 5.729998 \\
\hline & 1.109921 & -3.076033 & 5.976378 \\
\hline & 0.666952 & -3.161427 & 6.970124 \\
\hline & 0.826483 & -3.983320 & 4.977889 \\
\hline & 1.414115 & -3.850564 & 3.686819 \\
\hline & 2.281032 & -2.814472 & 3.412386 \\
\hline & 2.740273 & -2.697721 & 2.429191 \\
\hline & 1.172179 & -4.579627 & 2.911691 \\
\hline & 0.144324 & -4.812699 & 5.172282 \\
\hline & 3.453395 & -0.846771 & 4.133259 \\
\hline & 2.263944 & -1.106139 & 6.720114 \\
\hline & 2.416294 & 5.055056 & 13.545633 \\
\hline 7 & 1.444191 & 5.925031 & 12.333421 \\
\hline & 2.827092 & 7.193750 & 13.442188 \\
\hline | & 3.817840 & 7.127076 & 13.912623 \\
\hline & 2.094250 & 7.459746 & 14.217539 \\
\hline$y$ & 2.844783 & 7.999247 & 12.694429 \\
\hline & 5.673914 & 7.416172 & 1.674812 \\
\hline 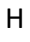 & 7.413774 & 7.410875 & 1.291739 \\
\hline & 6.763323 & 9.118497 & 2.485283 \\
\hline & 6.015648 & 9.337601 & 3.260232 \\
\hline 1 & 6.569358 & 9.768427 & 1.620373 \\
\hline & 7.760797 & 9.356471 & 2.879953 \\
\hline | & 7.247118 & 5.331459 & 11.592570 \\
\hline & 5.732117 & 4.931936 & 12.477808 \\
\hline & 5.826718 & 6.411936 & 11.521052 \\
\hline & 9.229933 & 6.229544 & 4.013577 \\
\hline
\end{tabular}

[Ni(2)]; $S=0$.

55

$\mathrm{XYZ}$ file generated by orca_plot on BaseName=HS

$\begin{array}{llll}\mathrm{Ni} & 5.681551 & 4.222761 & 7.136624 \\ \mathrm{O} & 6.940973 & 5.410427 & 6.454450 \\ \mathrm{O} & 5.913062 & 5.169930 & 8.722853 \\ \mathrm{~N} & 4.437274 & 3.060568 & 7.849119 \\ \mathrm{O} & 7.290272 & 3.876115 & 1.958780 \\ \mathrm{O} & 2.834874 & 2.937203 & 11.588771 \\ \mathrm{~N} & 5.486597 & 3.299771 & 5.550003 \\ \mathrm{C} & 4.020631 & 2.055168 & 6.948193 \\ \mathrm{C} & 5.297700 & 5.061725 & 9.845088 \\ \mathrm{C} & 3.931573 & 3.150545 & 9.068305 \\ \mathrm{H} & 3.162691 & 2.429434 & 9.359201 \\ \mathrm{C} & 4.608765 & 2.195977 & 5.641292 \\ \mathrm{C} & 7.404916 & 5.529176 & 5.262333 \\ \mathrm{C} & 6.083811 & 3.616918 & 4.413305\end{array}$

$\begin{array}{llll}\text { H } & 9.786165 & 6.069699 & 5.717850 \\ \text { H } & 8.529036 & 7.255131 & 5.273682 \\ \text { N } & 7.477375 & 2.125592 & 7.898572 \\ \mathrm{C} & 7.247235 & 1.362067 & 8.990126 \\ \mathrm{H} & 6.602141 & 1.801701 & 9.751625 \\ \mathrm{C} & 8.267524 & 1.594380 & 6.939121 \\ \mathrm{H} & 8.435177 & 2.223055 & 6.063939 \\ \mathrm{C} & 7.768679 & 0.092990 & 9.168412 \\ \mathrm{C} & 8.839264 & 0.336740 & 7.019382 \\ \mathrm{C} & 8.595627 & -0.477710 & 8.160874 \\ \mathrm{H} & 7.523085 & -0.452052 & 10.077626 \\ \mathrm{H} & 9.457009 & -0.012015 & 6.194364 \\ \mathrm{~N} & 5.287636 & 5.643612 & 7.136926 \\ \mathrm{C} & 4.214854 & 5.699611 & 6.319970 \\ \mathrm{H} & 3.686903 & 4.758612 & 6.157685 \\ \mathrm{C} & 5.926072 & 6.809793 & 7.380035 \\ \mathrm{H} & 6.777890 & 6.749554 & 8.058968 \\ \mathrm{C} & 3.780932 & 6.853220 & 5.690713 \\ \mathrm{C} & 5.569955 & 8.019009 & 6.810134 \\ \mathrm{C} & 4.488240 & 8.070515 & 5.889147 \\ \mathrm{H} & 2.908046 & 6.801377 & 5.043553 \\ \mathrm{H} & 6.143992 & 8.907456 & 7.062809 \\ \mathrm{~N} & 9.122127 & -1.726949 & 8.282046 \\ \mathrm{C} & 9.916719 & -2.297235 & 7.196286 \\ \mathrm{C} & 8.785510 & -2.556343 & 9.436982 \\ \mathrm{H} & 7.705229 & -2.771631 & 9.481186 \\ \mathrm{H} & 9.083814 & -2.067432 & 10.377127 \\ \mathrm{H} & 9.325158 & -3.504146 & 9.358241 \\ \mathrm{H} & 9.320447 & -2.419919 & 6.277206 \\ \mathrm{H} & 10.282217 & -3.280101 & 7.507095 \\ \mathrm{H} & 10.786229 & -1.662997 & 6.966944 \\ \mathrm{H} & 4.579772 & 10.776660 & 6.630035 \\ \mathrm{H} & 4.437147 & 11.262775 & 4.919893 \\ \mathrm{C} & 4.800605 & 10.478824 & 5.590956 \\ \mathrm{H} & 5.892194 & 10.406215 & 5.473406 \\ \mathrm{~N} & 4.150618 & 9.219296 & 5.238046 \\ \mathrm{H} & 2.045924 & 9.099586 & 4.900836 \\ \mathrm{H} & 2.991123 & 9.244869 & 4.350699 \\ & 3.070920 & 8.461973 & 3.582109\end{array}$




$\begin{array}{lrrr}\text { C } & 8.595863 & 6.041894 & 1.144944 \\ \text { C } & 2.286836 & -1.650013 & 4.176104 \\ \text { C } & 1.705536 & -1.800472 & 5.498013 \\ \text { C } & 0.815379 & -2.887386 & 5.743104 \\ \text { H } & 0.388116 & -2.986507 & 6.742073 \\ \text { C } & 0.514406 & -3.775410 & 4.735453 \\ \text { C } & 1.082675 & -3.625511 & 3.435039 \\ \text { C } & 1.948112 & -2.591340 & 3.159493 \\ \text { H } & 2.391863 & -2.462304 & 2.171093 \\ \text { H } & 0.824221 & -4.343187 & 2.654882 \\ \text { H } & -0.167430 & -4.605673 & 4.926368 \\ \text { N } & 3.136209 & -0.640492 & 3.891606 \\ \text { N } & 1.988593 & -0.934639 & 6.494003\end{array}$

[Ni(3)]; $S=0$.

$\begin{array}{cccc}\mathrm{H} & 6.714712 & 6.483283 & 10.537643 \\ \mathrm{H} & 5.970087 & 5.505951 & 11.851488 \\ \mathrm{H} & 5.038192 & 6.793778 & 11.086855 \\ \mathrm{H} & 9.338261 & 6.250429 & 4.623281 \\ \mathrm{H} & 8.679712 & 6.994126 & 6.122119 \\ \mathrm{H} & 8.039426 & 7.438572 & 4.508317 \\ \mathrm{H} & 2.996035 & 5.859915 & 13.886691 \\ \mathrm{H} & 3.218489 & 4.072976 & 13.964590 \\ \mathrm{H} & 1.752624 & 4.758969 & 13.187055 \\ \mathrm{H} & 9.063397 & 7.029732 & 1.101655 \\ \mathrm{H} & 7.774651 & 5.970333 & 0.420893 \\ \mathrm{H} & 9.334316 & 5.254854 & 0.947515\end{array}$

53

$\mathrm{XYZ}$ file generated by orca_plot on BaseName=HS

$\begin{array}{llll}\mathrm{Ni} & 5.703804 & 4.218097 & 7.147952 \\ \mathrm{O} & 6.944842 & 5.420600 & 6.461389 \\ \mathrm{O} & 5.901254 & 5.186674 & 8.724788 \\ \mathrm{~N} & 4.481304 & 3.039913 & 7.867949 \\ \mathrm{O} & 7.514924 & 3.681874 & 2.102456 \\ \mathrm{O} & 2.968323 & 2.802436 & 11.570194 \\ \mathrm{~N} & 5.552760 & 3.265876 & 5.574837 \\ \mathrm{C} & 4.061495 & 2.037435 & 6.962495 \\ \mathrm{C} & 5.289620 & 5.071009 & 9.848883 \\ \mathrm{C} & 3.991251 & 3.114059 & 9.092657 \\ \mathrm{H} & 3.252167 & 2.367417 & 9.397172 \\ \mathrm{C} & 4.661641 & 2.170584 & 5.660946 \\ \mathrm{C} & 7.427466 & 5.523931 & 5.274797 \\ \mathrm{C} & 6.185539 & 3.556433 & 4.451798 \\ \mathrm{H} & 6.014685 & 2.912492 & 3.584238 \\ \mathrm{C} & 7.068416 & 4.632875 & 4.220624 \\ \mathrm{C} & 4.300231 & 4.070435 & 10.083580 \\ \mathrm{C} & 3.177476 & 1.013605 & 7.228848 \\ \mathrm{H} & 2.712571 & 0.877938 & 8.204086 \\ \mathrm{C} & 3.523150 & 3.890669 & 11.340688 \\ \mathrm{C} & 2.844100 & 0.081793 & 6.209822 \\ \mathrm{C} & 4.356600 & 1.282365 & 4.651317 \\ \mathrm{H} & 4.789838 & 1.353018 & 3.654681 \\ \mathrm{C} & 7.571579 & 4.694497 & 2.820509 \\ \mathrm{C} & 3.443546 & 0.221117 & 4.892450 \\ \mathrm{C} & 8.447631 & 6.623642 & 5.146433\end{array}$

[Ni(4)]; $S=0$.

61

XYZ file generated by orca_plot on BaseName=HS

$\begin{array}{llll}\mathrm{Ni} & 5.888842 & 4.058901 & 7.223131 \\ \mathrm{O} & 7.226179 & 5.175839 & 6.569132 \\ \mathrm{O} & 6.208507 & 4.927320 & 8.839455 \\ \mathrm{~N} & 4.575382 & 2.953679 & 7.910932 \\ \mathrm{O} & 7.550300 & 3.818339 & 2.030890 \\ \mathrm{O} & 3.074960 & 2.891479 & 11.766114 \\ \mathrm{~N} & 5.624133 & 3.193756 & 5.611326 \\ \mathrm{C} & 4.122834 & 1.975048 & 6.993892 \\ \mathrm{C} & 5.549646 & 4.816107 & 9.927633 \\ \mathrm{C} & 4.051282 & 3.063326 & 9.117005 \\ \mathrm{H} & 3.229541 & 2.393209 & 9.383513 \\ \mathrm{C} & 4.710332 & 2.115475 & 5.691589\end{array}$

$\begin{array}{llll}\text { C } & 5.775983 & 6.046857 & 10.887227 \\ \text { C } & 3.329872 & 5.023749 & 12.323766 \\ \text { C } & 8.089486 & 5.981677 & 2.218117 \\ \text { C } & 2.281093 & -1.628787 & 4.169405 \\ \text { C } & 1.686245 & -1.770668 & 5.486476 \\ \text { C } & 0.772686 & -2.840120 & 5.722069 \\ \text { H } & 0.335697 & -2.932857 & 6.717428 \\ \text { C } & 0.461969 & -3.719385 & 4.709827 \\ \text { C } & 1.043486 & -3.577888 & 3.414217 \\ \text { C } & 1.931749 & -2.560924 & 3.147917 \\ \text { H } & 2.385784 & -2.438599 & 2.163369 \\ \text { H } & 0.776700 & -4.288364 & 2.630318 \\ \text { H } & -0.238058 & -4.536104 & 4.893191 \\ \text { N } & 3.152110 & -0.635549 & 3.893868 \\ \text { N } & 1.977984 & -0.912658 & 6.486442 \\ \text { H } & 6.708672 & 6.495814 & 10.529207 \\ \text { H } & 5.952928 & 5.562223 & 11.854365 \\ \text { H } & 5.041556 & 6.850798 & 11.040116 \\ \text { H } & 9.361573 & 6.274600 & 4.650225 \\ \text { H } & 8.696175 & 6.986077 & 6.149464 \\ \text { H } & 8.045256 & 7.464711 & 4.563528 \\ \text { H } & 3.214302 & 5.997671 & 11.833382 \\ \text { H } & 4.190428 & 5.091973 & 13.006318 \\ \text { H } & 2.439878 & 4.801732 & 12.924480 \\ \text { H } & 8.031483 & 5.891107 & 1.126583 \\ \text { H } & 9.144315 & 6.137573 & 2.489372 \\ \text { H } & 7.524627 & 6.861224 & 2.550653\end{array}$

$\begin{array}{llll}\text { C } & 7.632447 & 5.290431 & 5.363802 \\ \text { C } & 6.193699 & 3.529130 & 4.469852 \\ \text { H } & 5.921295 & 2.965564 & 3.573826 \\ \text { C } & 7.137392 & 4.560332 & 4.269508 \\ \text { C } & 4.440433 & 3.975250 & 10.121936 \\ \text { C } & 3.228172 & 0.961458 & 7.260093 \\ \text { H } & 2.782436 & 0.811761 & 8.242145 \\ \text { C } & 3.601045 & 3.919873 & 11.352682 \\ \text { C } & 2.867697 & 0.052221 & 6.229509 \\ \text { C } & 4.385961 & 1.246982 & 4.672472 \\ \text { H } & 4.821558 & 1.315122 & 3.677025 \\ \text { C } & 7.539900 & 4.737248 & 2.845122 \\ \text { C } & 3.456017 & 0.199886 & 4.909424 \\ \text { C } & 8.824168 & 6.279699 & 5.257624\end{array}$




$\begin{array}{llll}\text { F } & 9.790775 & 5.828840 & 4.417316 \\ \text { F } & 8.425361 & 7.508043 & 4.839342 \\ \text { F } & 9.404353 & 6.443089 & 6.470421 \\ \text { C } & 6.185015 & 5.654005 & 11.069247 \\ \text { F } & 6.147418 & 5.005752 & 12.262152 \\ \text { F } & 7.488408 & 5.900864 & 10.796774 \\ \text { F } & 5.574180 & 6.857738 & 11.214879 \\ \text { O } & 3.434136 & 5.132377 & 11.908658 \\ \text { C } & 2.679952 & 5.177199 & 13.168269 \\ \text { O } & 7.825447 & 6.014489 & 2.544090 \\ \text { C } & 8.312364 & 6.273731 & 1.183055 \\ \text { C } & 2.268051 & -1.626710 & 4.174344 \\ \text { C } & 1.681871 & -1.776747 & 5.495995 \\ \text { C } & 0.757249 & -2.837948 & 5.726730 \\ \text { H } & 0.326841 & -2.937208 & 6.724150 \\ \text { C } & 0.429253 & -3.700886 & 4.706631 \\ \text { C } & 1.002684 & -3.551666 & 3.407561 \\ \text { C } & 1.900196 & -2.542769 & 3.144739\end{array}$

[Ni(4)]; $S=1$.

$$
61
$$

XYZ file generated by orca_plot on BaseName=HS

$\begin{array}{lccc}\mathrm{Ni} & 6.049021 & 4.071541 & 7.282922 \\ \mathrm{O} & 7.484019 & 5.155515 & 6.524946 \\ \mathrm{O} & 6.294663 & 4.958883 & 9.000975 \\ \mathrm{~N} & 4.639932 & 2.946438 & 7.976635 \\ \mathrm{O} & 7.683231 & 3.806738 & 1.977367 \\ \mathrm{O} & 3.152249 & 2.858178 & 11.865068 \\ \mathrm{~N} & 5.731235 & 3.178802 & 5.599571 \\ \mathrm{C} & 4.185955 & 2.007404 & 7.028836 \\ \mathrm{C} & 5.612789 & 4.789438 & 10.060941 \\ \mathrm{C} & 4.082833 & 3.051652 & 9.171413 \\ \mathrm{H} & 3.226812 & 2.416163 & 9.423443 \\ \mathrm{C} & 4.783541 & 2.141623 & 5.711122 \\ \mathrm{C} & 7.858380 & 5.196673 & 5.311259 \\ \mathrm{C} & 6.268989 & 3.506553 & 4.436291 \\ \mathrm{H} & 5.939462 & 2.992347 & 3.527137 \\ \mathrm{C} & 7.260424 & 4.494474 & 4.239055 \\ \mathrm{C} & 4.487776 & 3.943379 & 10.191414 \\ \mathrm{C} & 3.278418 & 0.999968 & 7.277516 \\ \mathrm{H} & 2.829312 & 0.854096 & 8.259149 \\ \mathrm{C} & 3.620438 & 3.894385 & 11.407226 \\ \mathrm{C} & 2.900095 & 0.091771 & 6.249395 \\ \mathrm{C} & 4.433834 & 1.270338 & 4.701325 \\ \mathrm{H} & 4.871832 & 1.329487 & 3.705743 \\ \mathrm{C} & 7.616401 & 4.709325 & 2.804391 \\ \mathrm{C} & 3.487715 & 0.232740 & 4.931247 \\ \mathrm{C} & 9.144466 & 6.050455 & 5.113402 \\ \mathrm{~F} & 9.955864 & 5.564495 & 4.135664 \\ \mathrm{~F} & 8.853439 & 7.345123 & 4.821469 \\ \mathrm{~F} & 9.872611 & 6.054235 & 6.256634 \\ \mathrm{C} & 6.201153 & 5.560187 & 11.277719 \\ \mathrm{HN}\end{array}$

\section{[Ni(4)(py)]; $S=1$.}

72

$\mathrm{XYZ}$ file generated by orca_plot on BaseName=HS

$$
\begin{array}{llll}
\mathrm{Ni} & 6.243710 & 3.762091 & 7.309228 \\
\mathrm{O} & 7.569837 & 5.004092 & 6.462469 \\
\mathrm{O} & 6.357622 & 4.784554 & 9.033989
\end{array}
$$

$\begin{array}{cccc}\text { H } & 2.348224 & -2.414432 & 2.158350 \\ \text { H } & 0.721299 & -4.250518 & 2.618452 \\ \text { H } & -0.278730 & -4.511631 & 4.885487 \\ \text { N } & 3.147757 & -0.641773 & 3.903139 \\ \text { N } & 1.992622 & -0.935493 & 6.502963 \\ \text { H } & 3.198863 & 4.539791 & 13.897539 \\ \text { H } & 1.681791 & 4.757512 & 12.982843 \\ \mathrm{C} & 2.622226 & 6.624136 & 13.606004 \\ \mathrm{H} & 3.629304 & 7.027869 & 13.776087 \\ \mathrm{H} & 2.059573 & 6.690845 & 14.547763 \\ \mathrm{H} & 2.112356 & 7.243939 & 12.855668 \\ \mathrm{H} & 7.514866 & 5.998637 & 0.479420 \\ \mathrm{H} & 9.179640 & 5.624042 & 1.001546 \\ \mathrm{C} & 8.666557 & 7.741883 & 1.104333 \\ \mathrm{H} & 7.790309 & 8.372587 & 1.307651 \\ \mathrm{H} & 9.025217 & 7.969306 & 0.090424 \\ \mathrm{H} & 9.462225 & 7.996143 & 1.817414\end{array}$

$\begin{array}{llll}F & 6.039276 & 4.894679 & 12.452979\end{array}$

F $\quad 7.534453 \quad 5.735606 \quad 11.108114$

$\begin{array}{llll}F & 5.649708 & 6.795343 & 11.410401\end{array}$

$\begin{array}{llll}\text { O } & 3.359163 & 5.123713 & 11.886113\end{array}$

$\begin{array}{llll}\text { C } & 2.562007 & 5.188806 & 13.118926\end{array}$

$\begin{array}{llll}\text { O } & 7.779809 & 6.013793 & 2.520347\end{array}$

$\begin{array}{llll}\text { C } & 8.196456 & 6.337224 & 1.149279\end{array}$

$\begin{array}{llll}\text { C } & 2.274700 & -1.575997 & 4.196364\end{array}$

$\begin{array}{llll}\text { C } & 1.690221 & -1.720074 & 5.517092\end{array}$

$\begin{array}{llll}\text { C } & 0.755440 & -2.768526 & 5.750285\end{array}$

$\begin{array}{llll}\text { H } & 0.326415 & -2.863811 & 6.748642\end{array}$

$\begin{array}{llll}\text { C } & 0.414201 & -3.628634 & 4.729197\end{array}$

$\begin{array}{llll}\text { C } & 0.985111 & -3.484960 & 3.431340\end{array}$

$\begin{array}{llll}\text { C } & 1.894956 & -2.484348 & 3.167573\end{array}$

$\begin{array}{llll}H & 2.342022 & -2.360773 & 2.180219\end{array}$

$\begin{array}{llll}H & 0.694583 & -4.179089 & 2.641535\end{array}$

$\begin{array}{llll}\text { H } & -0.302507 & -4.431051 & 4.910236\end{array}$

$\begin{array}{llll}\mathrm{N} & 3.168790 & -0.599405 & 3.924501\end{array}$

$\begin{array}{llll}N & 2.014191 & -0.880884 & 6.526129\end{array}$

$\begin{array}{llll}H & 3.114000 & 4.656621 & 13.906229\end{array}$

$\begin{array}{llll}\text { H } & 1.613672 & 4.664188 & 12.939689\end{array}$

$\begin{array}{llll}\text { C } & 2.357021 & 6.650994 & 13.445640\end{array}$

$\begin{array}{llll}\text { H } & 3.316744 & 7.160551 & 13.605609\end{array}$

$\begin{array}{llll}\mathrm{H} & 1.765214 & 6.731393 & 14.368641\end{array}$

$\begin{array}{llll}H & 1.811480 & 7.162691 & 12.640628\end{array}$

$\begin{array}{llll}H & 7.437406 & 5.941364 & 0.460977\end{array}$

$\begin{array}{llll}H & 9.148707 & 5.823757 & 0.955121\end{array}$

$\begin{array}{llll}\text { C } & 8.324230 & 7.841205 & 1.060711\end{array}$

$\begin{array}{llll}\text { H } & 7.364209 & 8.332747 & 1.271103\end{array}$

$\begin{array}{llll}\text { H } & 8.633614 & 8.115703 & 0.042390\end{array}$

H $\quad 9.079635 \quad 8.217235 \quad 1.763786$ 


$\begin{array}{llll}\text { C } & 5.533798 & 4.738126 & 9.997607 \\ \text { C } & 3.904630 & 3.142724 & 8.994355 \\ \text { H } & 2.933539 & 2.657654 & 9.150479 \\ \text { C } & 4.787995 & 2.014563 & 5.663533 \\ \text { C } & 7.812134 & 5.131667 & 5.223549 \\ \text { C } & 6.146703 & 3.484749 & 4.375057 \\ \text { H } & 5.710641 & 3.063018 & 3.461785 \\ \text { C } & 7.135030 & 4.484951 & 4.162539 \\ \text { C } & 4.304114 & 4.037963 & 10.023677 \\ \text { C } & 3.263376 & 0.896709 & 7.233384 \\ \text { H } & 2.813205 & 0.770422 & 8.217744 \\ \text { C } & 3.290430 & 4.175828 & 11.105295 \\ \text { C } & 2.924086 & -0.044751 & 6.225172 \\ \text { C } & 4.492445 & 1.094570 & 4.678589 \\ \text { H } & 4.979468 & 1.120013 & 3.704287 \\ \text { C } & 7.362381 & 4.777210 & 2.720188 \\ \text { C } & 3.550553 & 0.058826 & 4.917204 \\ \text { C } & 9.046440 & 6.051856 & 4.964697 \\ \text { F } & 9.813938 & 5.635444 & 3.921122 \\ \text { F } & 8.686165 & 7.343352 & 4.742465 \\ \text { F } & 9.851566 & 6.050855 & 6.057373 \\ \text { C } & 6.068821 & 5.490433 & 11.255971 \\ \text { F } & 5.693053 & 4.910424 & 12.427871 \\ \text { F } & 7.426589 & 5.487498 & 11.244302 \\ \text { F } & 5.678150 & 6.791996 & 11.277680 \\ \text { O } & 3.184414 & 5.445172 & 11.546491 \\ \text { C } & 2.273539 & 5.671214 & 12.673257 \\ \text { O } & 7.560892 & 6.088825 & 2.483927 \\ \text { C } & 7.892374 & 6.459072 & 1.103496 \\ \text { C } & 2.382198 & -1.793692 & 4.211493 \\ \text { C } & 1.759475 & -1.899623 & 5.519003 \\ \text { C } & 0.826921 & -2.951231 & 5.758196\end{array}$

\section{[Ni(4)(py) $\left.)_{2}\right] S=1$.}

83

$\mathrm{XYZ}$ file generated by orca_plot on BaseName=HS

$\begin{array}{cccc}\mathrm{Ni} & 6.368037 & 3.692035 & 7.377098 \\ \mathrm{O} & 7.990599 & 4.610578 & 6.498689 \\ \mathrm{O} & 6.632327 & 4.364659 & 9.311045 \\ \mathrm{~N} & 4.710709 & 2.745293 & 7.959164 \\ \mathrm{O} & 6.880195 & 4.385487 & 1.871271 \\ \mathrm{O} & 2.244355 & 3.824248 & 11.112397 \\ \mathrm{~N} & 5.870594 & 2.920004 & 5.605285 \\ \mathrm{C} & 4.313860 & 1.773534 & 7.037117 \\ \mathrm{C} & 5.665004 & 4.545207 & 10.107732 \\ \mathrm{C} & 3.951529 & 3.144852 & 8.949965 \\ \mathrm{H} & 2.920756 & 2.773387 & 9.020769 \\ \mathrm{C} & 4.957343 & 1.869019 & 5.733128 \\ \mathrm{C} & 8.008924 & 4.950866 & 5.278468 \\ \mathrm{C} & 6.182925 & 3.457747 & 4.451941 \\ \mathrm{H} & 5.654924 & 3.129800 & 3.547380 \\ \mathrm{C} & 7.137104 & 4.498521 & 4.256397 \\ \mathrm{C} & 4.334111 & 4.081341 & 9.952733 \\ \mathrm{C} & 3.423744 & 0.752605 & 7.300041 \\ \mathrm{H} & 2.969800 & 0.636486 & 8.284459 \\ \mathrm{C} & 3.203771 & 4.529282 & 10.803053 \\ \mathrm{C} & 3.101133 & -0.199990 & 6.295498 \\ \mathrm{C} & 4.677505 & 0.939522 & 4.752290 \\ \mathrm{H} & 5.178673 & 0.966838 & 3.784701\end{array}$

$\begin{array}{cccc}\text { H } & 0.369014 & -3.017275 & 6.746230 \\ \mathrm{C} & 0.524007 & -3.847171 & 4.757901 \\ \mathrm{C} & 1.132757 & -3.740644 & 3.471708 \\ \mathrm{C} & 2.040564 & -2.741643 & 3.202554 \\ \mathrm{H} & 2.515432 & -2.645407 & 2.224983 \\ \mathrm{H} & 0.871292 & -4.463252 & 2.697169 \\ \mathrm{H} & -0.191138 & -4.650079 & 4.943734 \\ \mathrm{~N} & 3.273098 & -0.818566 & 3.933177 \\ \mathrm{~N} & 2.044223 & -1.025193 & 6.507372 \\ \mathrm{H} & 2.580774 & 5.014368 & 13.499342 \\ \mathrm{H} & 1.261057 & 5.383856 & 12.357606 \\ \mathrm{C} & 2.365264 & 7.136443 & 13.039293 \\ \mathrm{H} & 3.385157 & 7.405034 & 13.345901 \\ \mathrm{H} & 1.686528 & 7.339954 & 13.879681 \\ \mathrm{H} & 2.068838 & 7.774641 & 12.195308 \\ \mathrm{H} & 7.044263 & 6.181711 & 0.462476 \\ \mathrm{H} & 8.769885 & 5.873884 & 0.793583 \\ \mathrm{C} & 8.159794 & 7.947766 & 1.087740 \\ \mathrm{H} & 7.276829 & 8.512365 & 1.418409 \\ \mathrm{H} & 8.401148 & 8.258972 & 0.061509 \\ \mathrm{H} & 9.008851 & 8.204454 & 1.735447 \\ \mathrm{~N} & 7.522053 & 2.298437 & 7.813108 \\ \mathrm{C} & 7.436307 & 1.709121 & 9.023799 \\ \mathrm{H} & 6.678658 & 2.096596 & 9.704007 \\ \mathrm{C} & 8.435180 & 1.852454 & 6.925798 \\ \mathrm{H} & 8.461124 & 2.352073 & 5.957772 \\ \mathrm{C} & 8.266295 & 0.653590 & 9.390552 \\ \mathrm{C} & 9.298924 & 0.801452 & 7.222048 \\ \mathrm{C} & 9.212664 & 0.187833 & 8.474381 \\ \mathrm{H} & 8.161031 & 0.205306 & 10.377802 \\ \mathrm{H} & 10.019344 & 0.470935 & 6.474460 \\ \mathrm{H} & 9.872358 & -0.641472 & 8.731689\end{array}$

$\begin{array}{cccc}\text { C } & 7.112966 & 5.052679 & 2.878305 \\ \text { C } & 3.740389 & -0.102057 & 4.990782 \\ \text { C } & 9.210661 & 5.894146 & 4.958916 \\ \text { F } & 9.762049 & 5.681044 & 3.732610 \\ \text { F } & 8.865331 & 7.206617 & 5.049231 \\ \text { F } & 10.204999 & 5.690897 & 5.863628 \\ \text { C } & 6.118883 & 5.271928 & 11.412367 \\ \text { F } & 5.422021 & 4.900627 & 12.520817 \\ \text { F } & 7.421825 & 4.975141 & 11.667626 \\ \text { F } & 6.042493 & 6.624437 & 11.293673 \\ \text { O } & 3.306454 & 5.833891 & 11.132340 \\ \text { C } & 2.296181 & 6.358180 & 12.051990 \\ \text { O } & 7.292961 & 6.388889 & 2.860187 \\ \text { C } & 7.371389 & 7.018522 & 1.540836 \\ \text { C } & 2.586825 & -1.967211 & 4.288709 \\ \text { C } & 1.953992 & -2.067827 & 5.590205 \\ \text { C } & 1.025169 & -3.122463 & 5.828464 \\ \text { H } & 0.559402 & -3.184470 & 6.813195 \\ \text { C } & 0.734492 & -4.027196 & 4.831657 \\ \text { C } & 1.352953 & -3.925592 & 3.550469 \\ \text { C } & 2.258341 & -2.923040 & 3.283246 \\ \text { H } & 2.740629 & -2.830499 & 2.308853 \\ \text { H } & 1.101736 & -4.653989 & 2.777858 \\ \text { H } & 0.021742 & -4.832353 & 5.017328 \\ \text { N } & 3.475793 & -0.988191 & 4.011718\end{array}$




$\begin{array}{cccc}\mathrm{N} & 2.225211 & -1.183814 & 6.575474 \\ \mathrm{H} & 2.307199 & 5.744463 & 12.964109 \\ \mathrm{H} & 1.310770 & 6.255997 & 11.576712 \\ \mathrm{C} & 2.648822 & 7.803274 & 12.330411 \\ \mathrm{H} & 3.639843 & 7.884304 & 12.797302 \\ \mathrm{H} & 1.905564 & 8.230676 & 13.018821 \\ \mathrm{H} & 2.644838 & 8.395056 & 11.405050 \\ \mathrm{H} & 6.425257 & 6.832362 & 1.013492 \\ \mathrm{H} & 8.183598 & 6.537761 & 0.976482 \\ \mathrm{C} & 7.621706 & 8.494592 & 1.760277 \\ \mathrm{H} & 6.808938 & 8.949985 & 2.342716 \\ \mathrm{H} & 7.674382 & 9.000356 & 0.785984 \\ \mathrm{H} & 8.570613 & 8.660317 & 2.288331 \\ \mathrm{~N} & 7.540354 & 2.002317 & 7.809195 \\ \mathrm{C} & 7.340774 & 1.307004 & 8.946896 \\ \mathrm{H} & 6.642671 & 1.741969 & 9.661299 \\ \mathrm{C} & 8.390740 & 1.504109 & 6.889482 \\ \mathrm{H} & 8.520336 & 2.095117 & 5.983220\end{array}$

\section{[Ni(4)(CN-py)]; $S=1$.}

73

Coordinates from ORCA-job HS

$\begin{array}{lccc}\mathrm{Ni} & 6.250188 & 3.742048 & 7.303539 \\ \mathrm{O} & 7.577159 & 4.981393 & 6.458927 \\ \mathrm{O} & 6.374027 & 4.753552 & 9.031265 \\ \mathrm{~N} & 4.585778 & 2.860413 & 7.894713 \\ \mathrm{O} & 7.296890 & 3.927714 & 1.830192 \\ \mathrm{O} & 2.591680 & 3.242742 & 11.498445 \\ \mathrm{~N} & 5.719495 & 3.046248 & 5.534496 \\ \mathrm{C} & 4.155025 & 1.904026 & 6.961761 \\ \mathrm{C} & 5.549009 & 4.713030 & 9.994890 \\ \mathrm{C} & 3.910886 & 3.129239 & 8.989940 \\ \mathrm{H} & 2.937426 & 2.648956 & 9.145465 \\ \mathrm{C} & 4.783343 & 2.006647 & 5.654324 \\ \mathrm{C} & 7.812115 & 5.120437 & 5.219207 \\ \mathrm{C} & 6.141826 & 3.480769 & 4.368667 \\ \mathrm{H} & 5.698837 & 3.066536 & 3.455435 \\ \mathrm{C} & 7.130998 & 4.480335 & 4.157509 \\ \mathrm{C} & 4.315801 & 4.020355 & 10.020351 \\ \mathrm{C} & 3.255863 & 0.890716 & 7.222406 \\ \mathrm{H} & 2.806405 & 0.763013 & 8.206902 \\ \mathrm{C} & 3.302999 & 4.162542 & 11.103112 \\ \mathrm{C} & 2.910198 & -0.044618 & 6.210718 \\ \mathrm{C} & 4.481910 & 1.092480 & 4.665892 \\ \mathrm{H} & 4.967565 & 1.119751 & 3.690968 \\ \mathrm{C} & 7.353817 & 4.777962 & 2.714836 \\ \mathrm{C} & 3.534996 & 0.060856 & 4.902293 \\ \mathrm{C} & 9.039869 & 6.049674 & 4.961650 \\ \mathrm{~F} & 9.806226 & 5.641950 & 3.914156 \\ \mathrm{~F} & 8.669960 & 7.339079 & 4.745958 \\ \mathrm{~F} & 9.847626 & 6.048958 & 6.052165 \\ \mathrm{C} & 6.087674 & 5.464382 & 11.252215 \\ \mathrm{~F} & 5.708335 & 4.887619 & 12.424226 \\ \mathrm{~F} & 7.445240 & 5.454467 & 11.240534 \\ \mathrm{~F} & 5.702796 & 6.767316 & 11.271242 \\ \mathrm{O} & 3.201499 & 5.432623 & 11.541825 \\ \mathrm{C} & 2.290978 & 5.664396 & 12.668113 \\ \mathrm{O} & 7.549898 & 6.089854 & 2.481829 \\ \mathrm{C} & 7.875314 & 6.464757 & 1.100857\end{array}$

$\begin{array}{llll}\text { C } & 7.979781 & 0.096449 & 9.205814\end{array}$

$\begin{array}{llll}\text { C } & 9.069063 & 0.300830 & 7.071871\end{array}$

$\begin{array}{llll}\text { C } & 8.856857 & -0.421378 & 8.249247\end{array}$

$\begin{array}{llll}\text { H } & 7.782709 & -0.429728 & 10.139690\end{array}$

$\begin{array}{llll}H & 9.744218 & -0.061613 & 6.296768\end{array}$

$\begin{array}{llll}H & 9.366429 & -1.370484 & 8.418344\end{array}$

$\begin{array}{llll}\mathrm{N} & 5.313794 & 5.478571 & 7.002667\end{array}$

$\begin{array}{llll}\text { C } & 4.114396 & 5.482716 & 6.389467\end{array}$

$\begin{array}{llll}\text { H } & 3.710514 & 4.510363 & 6.107267\end{array}$

$\begin{array}{llll}\text { C } & 5.855867 & 6.656107 & 7.372280\end{array}$

$\begin{array}{llll}\text { H } & 6.826861 & 6.602158 & 7.863882\end{array}$

$\begin{array}{llll}\text { C } & 3.415494 & 6.657091 & 6.121609\end{array}$

$\begin{array}{llll}\text { C } & 5.221017 & 7.874491 & 7.142000\end{array}$

C $3.978383 \quad 7.877285 \quad 6.504407$

$\begin{array}{llll}\text { H } & 2.448333 & 6.608769 & 5.622414\end{array}$

$\begin{array}{llll}\text { H } & 5.698142 & 8.801423 & 7.458794\end{array}$

$\begin{array}{llll}\text { H } & 3.457384 & 8.815061 & 6.308505\end{array}$

$\begin{array}{lccc}\mathrm{C} & 2.355579 & -1.782142 & 4.190866 \\ \mathrm{C} & 1.734406 & -1.890093 & 5.499265 \\ \mathrm{C} & 0.796424 & -2.937550 & 5.735763 \\ \mathrm{H} & 0.339816 & -3.005178 & 6.724264 \\ \mathrm{C} & 0.486929 & -3.827366 & 4.732201 \\ \mathrm{C} & 1.094115 & -3.718836 & 3.445271 \\ \mathrm{C} & 2.006943 & -2.723950 & 3.178520 \\ \mathrm{H} & 2.480636 & -2.626319 & 2.200545 \\ \mathrm{H} & 0.827164 & -4.436743 & 2.668263 \\ \mathrm{H} & -0.232343 & -4.627077 & 4.915790 \\ \mathrm{~N} & 3.251137 & -0.810938 & 3.915041 \\ \mathrm{~N} & 2.025432 & -1.021416 & 6.490516 \\ \mathrm{H} & 2.596567 & 5.008853 & 13.495807 \\ \mathrm{H} & 1.277871 & 5.378952 & 12.352866 \\ \mathrm{C} & 2.387032 & 7.130307 & 13.030008 \\ \mathrm{H} & 3.407719 & 7.396783 & 13.335811 \\ \mathrm{H} & 1.709023 & 7.338022 & 13.869936 \\ \mathrm{H} & 2.092263 & 7.767048 & 12.184352 \\ \mathrm{H} & 7.025708 & 6.186213 & 0.462395 \\ \mathrm{H} & 8.753558 & 5.882948 & 0.786750 \\ \mathrm{C} & 8.138172 & 7.954212 & 1.088063 \\ \mathrm{H} & 7.254668 & 8.515349 & 1.423128 \\ \mathrm{H} & 8.375037 & 8.268568 & 0.061753 \\ \mathrm{H} & 8.988768 & 8.211847 & 1.733358 \\ \mathrm{~N} & 7.523328 & 2.274038 & 7.799045 \\ \mathrm{C} & 7.432246 & 1.676972 & 9.005184 \\ \mathrm{H} & 6.677906 & 2.060824 & 9.690216 \\ \mathrm{C} & 8.434528 & 1.831740 & 6.908385 \\ \mathrm{H} & 8.467138 & 2.336687 & 5.943996 \\ \mathrm{C} & 8.249826 & 0.615705 & 9.371500 \\ \mathrm{C} & 9.294137 & 0.776841 & 7.188025 \\ \mathrm{C} & 9.197632 & 0.154115 & 8.443415 \\ \mathrm{H} & 8.145075 & 0.158193 & 10.353303 \\ \mathrm{H} & 10.015057 & 0.446546 & 6.442509 \\ \mathrm{C} & 10.054507 & -0.940951 & 8.773098 \\ \mathrm{~N} & 10.751679 & -1.835390 & 9.042383\end{array}$




\section{$\left[\mathrm{Ni}(4)(\mathrm{CN}-\mathrm{py})_{2}\right] ; S=1$.}

85

Coordinates from ORCA-job HS

\begin{tabular}{|c|c|c|c|}
\hline $\mathrm{Vi}$ & 6.417456 & 3.647795 & 7.359671 \\
\hline O & 8.059313 & 4.526776 & 6.471297 \\
\hline & 6.682598 & 4.322838 & 9.287379 \\
\hline & 4.745417 & 2.723020 & 7.940398 \\
\hline & 6.778293 & 4.546616 & 1.875012 \\
\hline & 2.274870 & 3.912198 & 11.066310 \\
\hline & 5.895485 & 2.887145 & 5.588019 \\
\hline & 4.349031 & 1.740701 & 7.027519 \\
\hline & 5.702797 & 4.548387 & 10.061133 \\
\hline & 3.983799 & 3.143672 & 8.920226 \\
\hline 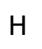 & 2.951284 & 2.777680 & 8.993511 \\
\hline & 4.985821 & 1.833043 & 5.721307 \\
\hline 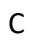 & 8.052376 & 4.88 & 5.254461 \\
\hline & 6.175994 & 3.453712 & 4.440133 \\
\hline - & 5.618881 & 3.154584 & 3.542914 \\
\hline & 7.132537 & 4.492343 & 4.253372 \\
\hline $\mathcal{L}$ & 4.368886 & 4.102717 & 9.900456 \\
\hline C & 3.466417 & 0.717251 & 7.301158 \\
\hline & 3.016458 & 0.604829 & 8.287831 \\
\hline 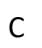 & 3.233044 & 4.598751 & 10.719021 \\
\hline 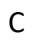 & 3.149053 & -0.244491 & 6.302658 \\
\hline$C$ & 4.709347 & 0.899042 & 4.744969 \\
\hline $\mathrm{H}$ & 5.207859 & 0.925768 & 3.775874 \\
\hline & 7.047425 & 5.138312 & 2.917903 \\
\hline C & 3.781870 & -0.149343 & 4.995067 \\
\hline$C$ & 9.286624 & 5.773858 & 4.906767 \\
\hline & 9.717555 & 5.640442 & 3.623365 \\
\hline F & 9.038920 & 7.090999 & 5.139184 \\
\hline & 10.335694 & 5.425373 & 5.698236 \\
\hline C & 6.143373 & 5.316580 & 11.344628 \\
\hline & 5.414784 & 4.999763 & 12.448428 \\
\hline $\mathrm{F}$ & 7.434694 & 5.009589 & 11.637870 \\
\hline & 6.090422 & 6.664608 & 11.170661 \\
\hline 0 & 3.333782 & 5.922527 & 10.959870 \\
\hline C & 2.308516 & 6.512095 & 11.823354 \\
\hline 0 & 7.208936 & 6.474913 & 3.002421 \\
\hline$C$ & 7.206630 & 7.217329 & 1.740246 \\
\hline$C$ & 2.648326 & -2.032969 & 4.312069 \\
\hline$C$ & 2.020908 & -2.130346 & 5.616862 \\
\hline$C$ & 1.106661 & -3.194770 & 5.867706 \\
\hline $\mathrm{H}$ & 0.645127 & -3.254259 & 6.854511 \\
\hline 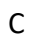 & 0.825669 & -4.112155 & 4.880009 \\
\hline
\end{tabular}

\section{[Ni(4)(DMAP)]; $S=1$.}

$$
80
$$

Coordinates from ORCA-job HS

$\begin{array}{cccc}\mathrm{Ni} & 6.257586 & 3.783741 & 7.308629 \\ \mathrm{O} & 7.578065 & 5.039221 & 6.465617 \\ \mathrm{O} & 6.361060 & 4.800008 & 9.040849 \\ \mathrm{~N} & 4.588853 & 2.898979 & 7.886468 \\ \mathrm{O} & 7.288729 & 4.019814 & 1.828877 \\ \mathrm{O} & 2.554260 & 3.281532 & 11.470454 \\ \mathrm{~N} & 5.734259 & 3.095950 & 5.533614 \\ \mathrm{C} & 4.180023 & 1.933279 & 6.955070 \\ \mathrm{C} & 5.529173 & 4.751496 & 9.996676\end{array}$

\begin{tabular}{llll}
$\mathrm{C}$ & 1.438863 & -4.013906 & 3.595825 \\
$\mathrm{C}$ & 2.329544 & -3.001878 & 3.316099 \\
$\mathrm{H}$ & 2.807728 & -2.911886 & 2.339521 \\
$\mathrm{H}$ & 1.195341 & -4.753079 & 2.831069 \\
$\mathrm{H}$ & 0.124890 & -4.925496 & 5.075047 \\
$\mathrm{~N}$ & 3.523851 & -1.045597 & 4.023809 \\
$\mathrm{~N}$ & 2.284843 & -1.235225 & 6.593550 \\
$\mathrm{H}$ & 2.350693 & 6.008160 & 12.799849 \\
$\mathrm{H}$ & 1.325143 & 6.317909 & 11.373538 \\
$\mathrm{C}$ & 2.607244 & 7.991685 & 11.927779 \\
$\mathrm{H}$ & 3.597730 & 8.164939 & 12.369779 \\
$\mathrm{H}$ & 1.853143 & 8.468517 & 12.569857 \\
$\mathrm{H}$ & 2.572350 & 8.470967 & 10.939937 \\
$\mathrm{H}$ & 6.247252 & 7.035515 & 1.235926 \\
$\mathrm{H}$ & 8.013843 & 6.821630 & 1.106578 \\
$\mathrm{C}$ & 7.406599 & 8.678242 & 2.078761 \\
$\mathrm{H}$ & 6.598318 & 9.046559 & 2.725522 \\
$\mathrm{H}$ & 7.403171 & 9.267269 & 1.151078 \\
$\mathrm{H}$ & 8.366905 & 8.838599 & 2.587450 \\
$\mathrm{~N}$ & 7.555005 & 1.943323 & 7.796405 \\
$\mathrm{C}$ & 7.356974 & 1.272083 & 8.948062 \\
$\mathrm{H}$ & 6.694264 & 1.738834 & 9.674855 \\
$\mathrm{C}$ & 8.365860 & 1.408493 & 6.862302 \\
$\mathrm{H}$ & 8.497147 & 1.982418 & 5.946327 \\
$\mathrm{C}$ & 7.953229 & 0.044839 & 9.214096 \\
$\mathrm{C}$ & 9.007314 & 0.187417 & 7.034991 \\
$\mathrm{C}$ & 8.790911 & -0.512372 & 8.233774 \\
$\mathrm{H}$ & 7.764067 & -0.466121 & 10.156460 \\
$\mathrm{H}$ & 9.651801 & -0.210468 & 6.253157 \\
$\mathrm{~N}$ & 5.395765 & 5.454725 & 6.993962 \\
$\mathrm{C}$ & 4.178982 & 5.480905 & 6.417862 \\
$\mathrm{H}$ & 3.754128 & 4.521043 & 6.127073 \\
$\mathrm{C}$ & 5.965348 & 6.616637 & 7.371243 \\
$\mathrm{H}$ & 6.949733 & 6.545077 & 7.831660 \\
$\mathrm{C}$ & 3.478444 & 6.660882 & 6.196065 \\
$\mathrm{C}$ & 5.344632 & 7.847746 & 7.192510 \\
$\mathrm{H}$ & 4.074190 & 7.869421 & 6.593320 \\
$\mathrm{H}$ & 2.496134 & 6.638456 & 5.728637 \\
$\mathrm{H}$ & 9.417835 & -1.777691 & 8.453037 \\
$\mathrm{H}$ & -2.811424 & 8.630437 \\
\hline & 9.110796 & 6.390164
\end{tabular}




\begin{tabular}{llll} 
C & 2.981614 & -0.045549 & 6.204846 \\
C & 4.542157 & 1.115290 & 4.666030 \\
H & 5.037249 & 1.146609 & 3.695907 \\
C & 7.341364 & 4.862288 & 2.721974 \\
C & 3.615681 & 0.064801 & 4.900611 \\
C & 9.020191 & 6.137202 & 4.971785 \\
F & 9.789662 & 5.749063 & 3.918765 \\
F & 8.633024 & 7.424358 & 4.767721 \\
F & 9.832519 & 6.140094 & 6.059475 \\
C & 6.057345 & 5.493850 & 11.264044 \\
F & 5.667069 & 4.912530 & 12.430832 \\
F & 7.415609 & 5.479455 & 11.265472 \\
F & 5.677891 & 6.798904 & 11.288147 \\
O & 3.173031 & 5.468705 & 11.522258 \\
C & 2.256390 & 5.701276 & 12.642391 \\
O & 7.526110 & 6.178351 & 2.497253 \\
C & 7.849106 & 6.564354 & 1.119432 \\
C & 2.483968 & -1.809767 & 4.192699 \\
C & 1.853714 & -1.922121 & 5.495643 \\
C & 0.937752 & -2.988796 & 5.731562 \\
H & 0.473815 & -3.059402 & 6.716498 \\
C & 0.657966 & -3.893675 & 4.732415 \\
C & 1.274201 & -3.780945 & 3.450543 \\
C & 2.166392 & -2.766915 & 3.184682 \\
H & 2.646687 & -2.665680 & 2.210229 \\
H & 1.031386 & -4.510757 & 2.676630 \\
H & -0.044315 & -4.708371 & 4.916047 \\
N & 3.360169 & -0.820160 & 3.917622 \\
N & 2.116314 & -1.039687 & 6.483385 \\
H & 2.553549 & 5.043160 & 13.471200 \\
IN & \\
\hline
\end{tabular}

[Ni(4)(DMAP) $\left.)_{2}\right] ;=1$.

99

Coordinates from ORCA-job HS

$\begin{array}{llll}\mathrm{Ni} & 6.409913 & 3.659401 & 7.351345 \\ \mathrm{O} & 8.020253 & 4.584112 & 6.449360 \\ \mathrm{O} & 6.617361 & 4.305588 & 9.338040 \\ \mathrm{~N} & 4.744525 & 2.707293 & 7.931768 \\ \mathrm{O} & 6.815061 & 4.382490 & 1.843249 \\ \mathrm{O} & 2.032304 & 4.274893 & 10.694790 \\ \mathrm{~N} & 5.900940 & 2.878758 & 5.586348 \\ \mathrm{C} & 4.394461 & 1.697630 & 7.037000 \\ \mathrm{C} & 5.593580 & 4.599983 & 10.020340 \\ \mathrm{C} & 3.922838 & 3.179616 & 8.836343 \\ \mathrm{H} & 2.879268 & 2.836191 & 8.849158 \\ \mathrm{C} & 5.037895 & 1.790643 & 5.732586 \\ \mathrm{C} & 8.005952 & 4.935064 & 5.233180 \\ \mathrm{C} & 6.191554 & 3.416246 & 4.428416 \\ \mathrm{H} & 5.665584 & 3.070445 & 3.528886 \\ \mathrm{C} & 7.120766 & 4.478248 & 4.223578 \\ \mathrm{C} & 4.258862 & 4.188766 & 9.781329 \\ \mathrm{C} & 3.550548 & 0.646078 & 7.326158 \\ \mathrm{H} & 3.099475 & 0.540495 & 8.313170 \\ \mathrm{C} & 3.083725 & 4.841970 & 10.400563 \\ \mathrm{C} & 3.285668 & -0.353268 & 6.350080 \\ \mathrm{C} & 4.810191 & 0.821253 & 4.777601 \\ \mathrm{H} & 5.314921 & 0.846779 & 3.811716 \\ \mathrm{C} & 7.060471 & 5.042800 & 2.852545 \\ \mathrm{C} & 3.928671 & -0.261922 & 5.045292\end{array}$

$\begin{array}{cccc}\text { H } & 1.243984 & 5.420650 & 12.320470 \\ \mathrm{C} & 2.355621 & 7.166198 & 13.008169 \\ \mathrm{H} & 3.375447 & 7.428022 & 13.320845 \\ \mathrm{H} & 1.673060 & 7.375239 & 13.844089 \\ \mathrm{H} & 2.069094 & 7.805766 & 12.161786 \\ \mathrm{H} & 7.000522 & 6.287429 & 0.478800 \\ \mathrm{H} & 8.729499 & 5.988640 & 0.799967 \\ \mathrm{C} & 8.106277 & 8.054995 & 1.116984 \\ \mathrm{H} & 7.220903 & 8.610385 & 1.456713 \\ \mathrm{H} & 8.341389 & 8.377857 & 0.092891 \\ \mathrm{H} & 8.956154 & 8.311152 & 1.763836 \\ \mathrm{~N} & 7.512216 & 2.305001 & 7.802201 \\ \mathrm{C} & 7.419619 & 1.684409 & 9.002015 \\ \mathrm{H} & 6.692211 & 2.091208 & 9.704322 \\ \mathrm{C} & 8.396753 & 1.805785 & 6.906905 \\ \mathrm{H} & 8.447301 & 2.310386 & 5.941699 \\ \mathrm{C} & 8.183030 & 0.588593 & 9.351188 \\ \mathrm{C} & 9.202586 & 0.714012 & 7.163558 \\ \mathrm{C} & 9.121588 & 0.052898 & 8.423064 \\ \mathrm{H} & 8.042159 & 0.148915 & 10.335642 \\ \mathrm{H} & 9.881446 & 0.374425 & 6.384901 \\ \mathrm{~N} & 9.889768 & -1.024633 & 8.720808 \\ \mathrm{C} & 10.795437 & -1.583709 & 7.718192 \\ \mathrm{C} & 9.727139 & -1.712713 & 10.001176 \\ \mathrm{H} & 8.717850 & -2.142557 & 10.104259 \\ \mathrm{H} & 9.906531 & -1.027115 & 10.843216 \\ \mathrm{H} & 10.457753 & -2.524706 & 10.058486 \\ \mathrm{H} & 10.243191 & -1.978496 & 6.850004 \\ \mathrm{H} & 11.360728 & -2.402587 & 8.172644 \\ \mathrm{H} & 11.511083 & -0.825368 & 7.366154\end{array}$

$\begin{array}{llll}C & 9.189281 & 5.895481 & 4.895020\end{array}$

$\begin{array}{llll}\text { F } & 9.720016 & 5.696867 & 3.655948\end{array}$

$\begin{array}{llll}\text { F } & 8.832834 & 7.203974 & 4.999889\end{array}$

F $\quad 10.205613 \quad 5.698896 \quad 5.777728$

$\begin{array}{llll}\text { C } \quad 5.953364 & 5.401858 & 11.306943\end{array}$

$\begin{array}{llll}\text { F } & 5.068470 & 5.238333 & 12.328838\end{array}$

$\begin{array}{llll}F & 7.160220 & 4.979856 & 11.778247\end{array}$

F $\quad 6.072984 \quad 6.734990 \quad 11.064214$

$\begin{array}{llll}\text { O } & 3.268625 & 6.177523 & 10.499236\end{array}$

$\begin{array}{llll}\text { C } & 2.189615 & 6.957907 & 11.098524\end{array}$

$\begin{array}{llll}\text { O } & 7.222010 & 6.382669 & 2.840666\end{array}$

C $\quad 7.260522 \quad 7.022010 \quad 1.524636$

$\begin{array}{llll}\text { C } & 2.887109 & -2.212807 & 4.400701\end{array}$

$\begin{array}{llll}\text { C } & 2.252010 & -2.306312 & 5.700767\end{array}$

$\begin{array}{llll}\text { C } & 1.386156 & -3.405556 & 5.970851\end{array}$

$\begin{array}{llll}\text { H } & 0.918255 & -3.461239 & 6.955022\end{array}$

$\begin{array}{llll}\text { C } & 1.157753 & -4.361076 & 5.005325\end{array}$

$\begin{array}{llll}\text { C } & 1.778315 & -4.266851 & 3.725068\end{array}$

$\begin{array}{llll}\text { C } & 2.623479 & -3.220611 & 3.427649\end{array}$

$\begin{array}{llll}\text { H } & 3.106745 & -3.133204 & 2.453209\end{array}$

$\begin{array}{llll}\text { H } & 1.577811 & -5.035886 & 2.977318\end{array}$

H $\quad 0.494135 \quad-5.201282 \quad 5.216010$

$\begin{array}{llll}\mathrm{N} & 3.720182 & -1.192976 & 4.095197\end{array}$

$\begin{array}{llll}N & 2.464287 & -1.374771 & 6.657022\end{array}$

$\begin{array}{llll}\mathrm{H} & 2.147805 & 6.720604 & 12.171886\end{array}$

$\begin{array}{llll}H & 1.240386 & 6.651682 & 10.637970\end{array}$

$\begin{array}{llll}\text { C } & 2.502938 & 8.416587 & 10.843437\end{array}$ 


$\begin{array}{llll}\mathrm{H} & 3.461839 & 8.701055 & 11.297326 \\ \mathrm{H} & 1.713472 & 9.044250 & 11.280240 \\ \mathrm{H} & 2.551641 & 8.615945 & 9.764064 \\ \mathrm{H} & 6.308812 & 6.818162 & 1.013863 \\ \mathrm{H} & 8.072104 & 6.563434 & 0.940989 \\ \mathrm{C} & 7.481481 & 8.502163 & 1.748247 \\ \mathrm{H} & 6.669753 & 8.935398 & 2.348915 \\ \mathrm{H} & 7.504073 & 9.014843 & 0.776395 \\ \mathrm{H} & 8.436276 & 8.686877 & 2.259182 \\ \mathrm{~N} & 7.574603 & 1.980234 & 7.778164 \\ \mathrm{C} & 7.352967 & 1.238698 & 8.887531 \\ \mathrm{H} & 6.685101 & 1.678334 & 9.628576 \\ \mathrm{C} & 8.397555 & 1.449804 & 6.844928 \\ \mathrm{H} & 8.563645 & 2.056674 & 5.954252 \\ \mathrm{C} & 7.914050 & -0.005992 & 9.107103 \\ \mathrm{C} & 9.008807 & 0.215542 & 6.967311 \\ \mathrm{C} & 8.773610 & -0.576699 & 8.126451 \\ \mathrm{H} & 7.676493 & -0.532650 & 10.029064 \\ \mathrm{H} & 9.652693 & -0.132174 & 6.162232 \\ \mathrm{~N} & 5.364130 & 5.448461 & 7.021371 \\ \mathrm{C} & 4.084555 & 5.479720 & 6.591054 \\ \mathrm{H} & 3.681916 & 4.539969 & 6.210825 \\ \mathrm{C} & 5.887481 & 6.614060 & 7.463801 \\ \mathrm{H} & 6.926003 & 6.574636 & 7.792307\end{array}$

$\begin{array}{cccc}\text { C } & 3.291368 & 6.612063 & 6.614991 \\ \text { C } & 5.182670 & 7.801277 & 7.519957 \\ \text { C } & 3.819344 & 7.830649 & 7.123078 \\ \text { H } & 2.271113 & 6.543918 & 6.245097 \\ \text { H } & 5.686284 & 8.691417 & 7.889247 \\ \text { N } & 9.335779 & -1.803961 & 8.286415 \\ \text { C } & 10.190058 & -2.363194 & 7.240529 \\ \text { C } & 9.034289 & -2.601337 & 9.473552 \\ \text { H } & 7.958332 & -2.828219 & 9.542890 \\ \text { H } & 9.343095 & -2.080965 & 10.393731 \\ \text { H } & 9.583191 & -3.544880 & 9.411913 \\ \text { H } & 9.635669 & -2.504445 & 6.298855 \\ \text { H } & 10.561429 & -3.336409 & 7.573381 \\ \text { H } & 11.055133 & -1.711751 & 7.042724 \\ \text { H } & 4.207170 & 10.151983 & 8.554641 \\ \text { H } & 2.912190 & 10.993379 & 7.680227 \\ \text { C } & 3.690634 & 10.229821 & 7.586716 \\ \text { H } & 4.418774 & 10.559112 & 6.825954 \\ \text { N } & 3.063090 & 8.960633 & 7.224589 \\ \text { H } & 1.107143 & 8.155437 & 7.169298 \\ \text { C } & 1.691237 & 8.972712 & 6.723052 \\ \text { H } & 1.222249 & 9.920514 & 7.006972 \\ \text { H } & 1.651037 & 8.874485 & 5.624791\end{array}$

